\title{
Parametric Correlations of Phase Shifts and Statistics of Time Delays in Quantum Chaotic Scattering: Crossover between Unitary and Orthogonal Symmetries
}

\author{
Yan V. Fyodorov ${ }^{a, c}$, Dmitry V. Savin ${ }^{b}$ and Hans-Jürgen Sommers ${ }^{a}$ \\ ${ }^{a}$ Fachbereich Physik, Universität-GH Essen, Essen 45117, Germany \\ ${ }^{b}$ Budker Institute of Nuclear Physics, 630090 Novosibirsk, Russia \\ ${ }^{c}$ Petersburg Nuclear Physics Institute, Gatchina 188350, Russia
}

(October 8, 2018)

We analyse universal statistical properties of phase shifts and time delays for open chaotic systems in the crossover regime of partly broken time-reversal invariance. In particular, we find that the distribution of the time delay shows $\tau^{-3 / 2}$ behavior for weakly open systems of any symmetry.

The energy-dependent scattering phase shifts $\theta_{a}(E)$ defined via the eigenvalues $\exp i \theta_{a} ; a=1, \ldots, M$ of the $M \times M$ unitary scattering matrix $\hat{S}(E)$ are important and frequently used characteristics of the process of quantum scattering. In particular, the derivatives of phase shifts over energy $\tau_{a}=\partial \theta_{a} / \partial E$ are related to the duration of a collision event. For example, the quantity $\tau_{W}=M^{-1} \sum_{a} \tau_{a}$ is the typical time delay due to scattering, the so-called Wigner-Smith time delay [1]. When some external parameters are taken into consideration (e.g. a magnetic field) the corresponding parametric variation of the phase shifts can as well be related to some observables [2].

Growing interest to the universal features [3] of quantum systems whose classical counterparts demonstrate chaotic dynamics attracted considerable attention to the process of quantum chaotic scattering, see [4.5] and references therein. From this point of view, different statistical characteristics of phase shifts and time delays were addressed in experiments on chaotic microwave reflection [6] as well as in several numerical studies of various models of quantum scattering in disordered and chaotic systems [7] 10 .

It is interesting to mention that for the case of only two open channels $M=2$ the phase shifts $\theta_{1,2}$ can simply be related to the phases of transmission and reflection coefficients, see e.g. [11]. The latter quantities are amenable to direct experimental measurements in quantum dots, see [12] and references therein. Another fact attributing additional interest to studies of time delay statistics is that it is intimately connected with the issue of mesoscopic fluctuations of dynamic admittances of microstructures [13.

One can extract statistical characteristics of the Smatrix exploiting a semiclassical periodic orbit expansion like that provided by the Gutzwiller trace formula, see examples of such calculations in [4] [6]. To proceed in this way, one has to employ some approximations (most frequently, the so called diagonal approximation). The resulting expressions provide an important insight into the problem. In particular, the semiclassical approximation for the time delay correlations at two different energies was derived by Eckhardt [14. However, the results obtained in such a way have a restricted domain of applicability; in particular, they fail to describe the system with only few open channels: $M \sim 1$.

A powerful alternative to the semiclassical methods in extracting the universal (i.e. generic and system independent ) statistical characteristics of the scattering matrix is provided by the random matrix approach. In particular, in the "Heidelberg variant" of this approach [15] one relates the scattering matrix $S(E)$ to the Hamiltonian of a closed counterpart of the open system. The latter Hamiltonian is considered to be a member of an ensemble of random matrices of appropriate global symmetry - an idea commonly accepted in the domain of Quantum Chaos. The use of random matrices gives one the possibility to apply the very powerful machinery of averaging developed by Efetov [16] and to calculate different correlation functions explicitly for any number of open channels and arbitrary coupling to continua.

When employing the Heidelberg method the actual calculation depends quite essentially on the symmetry of the underlying Hamiltonian. The simplest case to study corresponds to completely broken time reversal invariance (TRI) (systems in strong enough magnetic field), when the random matrix Hamiltonian $\hat{H}$ is taken from the Gaussian Unitary Ensemble (GUE). For such systems the statistics of phase shifts, delay times and resonance poles was thoroughly investigated by two of us recently [17], see a detailed exposition of the calculation in [18]. In the opposite case of fully preserved TRI when $H$ is a member of the Gaussian Orthogonal Ensemble (GOE) some aspects of time evolution of a chaotic system were considered in 20], the correlation function of WignerSmith time delays for two different energies was found in [19 and the distribution of time delays was obtained for the perfect coupling case in [13, see also 21]. Let us also mention the paper 22 addressing the issue of parametric correlations for $S-$ matrix elements.

In the present communication we extend the analysis of statistical properties of phase shifts and time delays to the whole crossover region of gradual breaking of the TRI. Different characteristics of chaotic and disordered systems in this crossover regime were under quite an in- 
tensive theoretical investigation recently 2325 .

Within the framework of random matrix theory, Hamiltonians of the closed chaotic systems under consideration are conveniently represented as [23,25]: $\hat{H}(y)=$ $\hat{H}_{S}+i \frac{y}{\sqrt{N}} \hat{H}_{A}$, where $\hat{H}_{S}$ is $N \times N$ GOE matrix and $\hat{H}_{A}$ is a real random antisymmetric matrix of the same dimension. For the sake of generality the symmetric matrix $\hat{H}_{S}$ is taken in the form [3]: $\hat{H}_{S}=\hat{H}_{S}^{(0)}+\frac{x}{\sqrt{N}} \hat{H}_{S}^{(1)}$. This form allows one to simulate the influence of such perturbations (e.g. a variation of the strength of scattering potential) which do not break the TRI. All elements of random matrices are independent and normalised in such a way that $\left\langle\operatorname{Tr}\left(\hat{H}_{S, A}^{(0,1)}\right)^{2}\right\rangle=N$.

In the limit $N \rightarrow \infty$ the crossover is driven by the parameter $y \in[0, \infty)$, with $y=\infty$ corresponding to completely broken TRI. Physically the parameter $y$ is proportional to the magnetix flux through the system $\Phi$. One may also notice that the typical shift of the levels due to the antisymmetric perturbation is $\delta E_{y} / \Delta \sim y^{2}$ [25], where $\Delta$ is the mean level spacing.

Within the framework of the Heidelberg approach 115] the coupling of the chaotic region to the incoming/outgoing waves is described with the help of the $M \times$ $N$ matrix $\hat{W}$ of amplitudes $W_{i a}, a=1,2, \ldots, M ; \quad i=$ $1, \ldots, N$, which couple the internal motion to $M$ open channels. In what follows we consider the case of arbitrary, but fixed $M$ whereas $N \rightarrow \infty$. Without much loss of generality these amplitudes can be chosen in a way ensuring that the average $S$-matrix is diagonal in the channel basis: $\left\langle S_{a b}\right\rangle=\delta_{a b}\left\langle S_{a a}\right\rangle$. The strength of coupling to continua is convenient to be characterized via the "sticking probabilities" (also called the "transmission coefficients") $T_{a}=1-\left|\left\langle S_{a a}\right\rangle\right|^{2}$ which are given for the present model by the following expression [15]:

$$
T_{a}^{-1}=\frac{1}{2}\left[1+\frac{\gamma_{a}+\gamma_{a}^{-1}}{2 \pi \nu(E)}\right] ; \quad \gamma_{a}=\pi \sum_{i} W_{i a}^{*} W_{i a}
$$

with $\nu(E)=\pi^{-1}\left(1-E^{2} / 4\right)^{1 / 2}$ being the density of states for the GOE matrices related to the local mean level spacing as $\Delta=(\nu N)^{-1}$. The quantity $T_{a}$ measures the part of the flux in channel $a$ that spends a substantial part of time in the interaction region $[15]$. We see that both limits $\gamma_{a} \rightarrow 0$ and $\gamma_{a} \rightarrow \infty$ equally correspond to the weak effective coupling regime $T_{a} \ll 1$ whereas the strongest coupling (at fixed energy $E$ ) corresponds to the value $\gamma_{a}=1$. The maximal possible coupling corresponding to the upper bound $T_{a}=1$ is achieved in the present model for an energy interval in the vicinity of the center $E=0$. Below we restrict our attention to this point of spectrum in order to present our final results in the most compact form. Moreover, we consider all channels to be statistically equivalent: $\gamma_{a}=\gamma$ for $a=1, \ldots, M$. Generalization to arbitrary $E$ and non-equivalent channels can easily be done, see 18 .

In the earlier work [17,18] it was shown that one can study very effectively the statistics of phase shifts $\theta_{a}$ considered modulo $2 \pi$. To this end we find it to be convenient to introduce the auxiliary "phases" $\phi$ related to the phase shifts $\theta$ as $\phi=\arctan \left\{\gamma^{-1} \tan (\theta / 2)\right\}$ and consider the density $\rho_{E, x, y}(\phi)=M^{-1} \sum_{a} \delta\left(\phi-\phi_{a}(E, x, y)\right)$.

The connected part of the correlation function of these densities is our main object of interest. It can be found performing calculations mutatis mutandis, similar to that presented in 17, 18] and it turns out to be dependent only on the difference $\phi=\phi_{1}-\phi_{2}$ :

$$
\begin{aligned}
& K_{\omega, x, y_{1}, y_{2}}^{\phi}(\phi)=\left\langle\rho_{E=0, x=0, y_{1}}\left(\phi_{1}\right) \rho_{E=\Omega, x, y_{2}}\left(\phi_{2}\right)\right\rangle_{c}= \\
& \operatorname{Re} \int_{-1}^{1} d \lambda \int_{1}^{\infty} d \lambda_{1} \int_{1}^{\infty} \frac{d \lambda_{2}}{\mathcal{R}^{2}} \mathcal{F}_{M}(\phi) e^{-\frac{x^{2}}{2}\left(2 \lambda_{1}^{2} \lambda_{2}^{2}-\lambda_{1}^{2}-\lambda_{2}^{2}-\lambda^{2}+1\right)} \times \\
& e^{-i \omega\left(\lambda_{1} \lambda_{2}-\lambda\right)+y_{1} y_{2}\left(\lambda_{1}^{2}-\lambda_{2}^{2}\right)-\frac{1}{2}\left(y_{1}^{2}+y_{2}^{2}\right)\left(\lambda_{1}^{2}+\lambda_{2}^{2}-\lambda^{2}-1\right)} \times \\
& \left\{\left(1-\lambda^{2}\right) \cosh \alpha-\left(\lambda_{1}^{2}-\lambda_{2}^{2}\right) \sinh \alpha+\right. \\
& \quad \mathcal{R}\left[\left(y_{1}^{2}+y_{2}^{2}\right)\left(\left(1-\lambda^{2}\right) \cosh \alpha+\left(\lambda_{2}^{2}-\lambda_{1}^{2}\right) \sinh \alpha\right)+\right. \\
& \left.\left.\quad 2 y_{1} y_{2}\left(\lambda_{1}^{2}+\lambda_{2}^{2}+\lambda^{2}-1\right) \sinh \alpha\right]\right\}
\end{aligned}
$$

where the "channel factor" is equal to

$\mathcal{F}_{M}(\phi)=-\frac{\partial^{2}}{\partial \phi^{2}}\left[\frac{(1+i \lambda \tan \phi)^{2}}{1+2 i \lambda_{1} \lambda_{2} \tan \phi-\tan ^{2} \phi\left(\lambda_{1}^{2}+\lambda_{2}^{2}-1\right)}\right]^{M / 2}$

and where $\mathcal{R}, \alpha, \omega$ denote $\mathcal{R}=\lambda_{1}^{2}+\lambda_{2}^{2}+\lambda^{2}-2 \lambda \lambda_{1} \lambda_{2}-$ $1, \quad \alpha=y_{1} y_{2}\left(1-\lambda^{2}\right)$ and $\omega=\pi \Omega / \Delta$.

The correlation function presented above is a very informative object. First of all, having it at our disposal it is a relatively easy task to show 18 that the correlation function of Wigner-Smith time delays: $K_{\omega, x, y_{1}, y_{2}}^{\tau}=$ $\left\langle\tau_{W}\left(E=0, x=0, y_{1}\right) \tau_{W}\left(E+\Omega, x, y_{2}\right)\right\rangle /\left\langle\tau_{W}(E)\right\rangle^{2}$ is given in the crossover regime by the same expression Eq.(2) provided one replaces the "channel factor" Eq.(3) by:

$\mathcal{F}_{M}^{\tau}=\left(\lambda_{1} \lambda_{2}-\lambda\right)^{2}\left[\frac{(g+\lambda)^{2}}{\left(g+\lambda_{1} \lambda_{2}\right)^{2}-\left(\lambda_{1}^{2}-1\right)\left(\lambda_{2}^{2}-1\right)}\right]^{M / 2}$,

here $g=2 / T-1$, with $T$ being the transmission coefficient introduced above.

Secondly, one can extract explicitly the general distribution function of the scaled partial delay times $\mathcal{P}\left(\tau_{s}\right)=$ $\left\langle\frac{1}{M} \sum_{a} \delta\left(\tau_{s}-\frac{\Delta}{2 \pi} \tau_{a}(y)\right)\right\rangle$ in the crossover regime:

$$
\begin{aligned}
& \mathcal{P}\left(\tau_{s}\right)=\frac{C_{M}}{\tau_{s}^{(M+5) / 2}} \int_{-1}^{1} d \lambda \int_{1}^{\infty} d \lambda_{2} \times \\
& \lambda_{2}^{\frac{M+3}{2}}\left(\lambda_{2}^{2}-1\right)^{\frac{1-M}{4}} e^{-2 y^{2}\left(\lambda_{2}^{2}-1\right)} \mathcal{J}_{1}\left(\lambda_{2}\right) \mathcal{J}_{2}\left(\lambda, \lambda_{2}\right)
\end{aligned}
$$

where $C_{M}=\left[(2 \pi)^{1 / 2} 2^{M / 2+1} \Gamma\left(\frac{M}{2}+1\right)\right]^{-1}$ and

$$
\begin{aligned}
& \mathcal{J}_{1}\left(\lambda_{2}\right)=\int_{0}^{\pi} d \psi v(\psi)^{\frac{M+1}{2}} e^{-\frac{\lambda_{2}^{2}}{\tau_{s}} v(\psi)} I_{\frac{M-1}{2}}\left[\frac{\lambda_{2} \sqrt{\lambda_{2}^{2}-1}}{\tau_{s}} v(\psi)\right] \\
& \mathcal{J}_{2}\left(\lambda, \lambda_{2}\right)=4 y^{2}\left[\left(1-\lambda^{2}\right) e^{-\beta}+\lambda_{2}^{2}\left(1-e^{-\beta}\right)\right]-\left(1-e^{-\beta}\right)
\end{aligned}
$$


where $v(\psi)=g-\sqrt{g^{2}-1} \cos \psi, \beta=2 y^{2}\left(1-\lambda^{2}\right)$ and $I_{p}(z)$ stands for the modified Bessel function.

The distribution Eq.(5) is valid for any number of open channels $M$ and any value of transmission coefficient $T$ and as such is quite complicated. To get a better understanding of its typical features it is reasonable to look separately at two limiting cases of strong/weak coupling to continua.

For the strong coupling regime $T=1$ (i.e. $g=1$ ) the $\psi$ - integration in Eq.(5) drops out, but the resulting expression is still quite cumbersome. However, one can easily find the long time asymptotics to be of the following form:

$$
\mathcal{P}\left(\tau_{s} \gg 1\right)=\left\{\begin{array}{cc}
U_{M}(y) \tau_{s}^{-(2+M)} & y>0 \\
(2 \pi)^{1 / 2} C_{M} e^{-\frac{1}{2 \tau_{s}}} \tau_{s}^{-(2+M / 2)} & y=0
\end{array}\right.
$$

where $U_{M}(y)$ is a rather complicated function of the symmetry breaking parameter $y$.

The second of this expressions holding for unbroken TRI is actually exact for arbitrary $\tau_{s}$ as can be seen performing the limit $y \rightarrow 0$ in the general Eq.(5) at $T=1$. This fact was first conjectured in 21] and derived for the particular case $M=1$ by another method in [13].

Eq.(6) demonstrates that the limits $y \rightarrow 0$ and $\tau \rightarrow \infty$ do not commute. To understand this phenomenon better it is instructive to consider the case of "weakly broken" TRI, $y \ll 1$, in more detail. A close inspection shows that for such regime there emerges one more relevant timescale $\tau_{y} \propto y^{-2} \gg 1$ such that for the domain $1 \ll \tau_{s} \ll \tau_{y}$ the distribution function $\mathcal{P}\left(\tau_{s}\right)$ shows the GOE-like behaviour: $\mathcal{P}\left(\tau_{s}\right) \propto \tau_{s}^{-(M / 2+2)}$, whereas at $\tau_{s} \gg \tau_{y}$ this behaviour changes to the GUE-like: $\mathcal{P}\left(\tau_{s}\right) \propto \tau_{y}^{M / 2} \tau_{s}^{-(M+2)}$.

We suggest the following transparent physical interpretation of the scale $\tau_{y}$ : this is just the time $\hbar / \delta E_{y}$ necessary by the Heisenberg uncertainty relation to resolve a typical shift $\delta E_{y}$ due to the TRI-breaking perturbation. If the particle dwells in the scattering domain for a time shorter than $\tau_{y}$ it can not "feel" the magnetic field effects and the corresponding asymptotics is GOE-like. However, for large enough times the particle resolves the effect of broken TRI whatever small is the magnetic field. This explains why the most distant asymptotics of the time delay distribution is always GUE-like, provided the magnetic field is not identically zero.

Let us now turn our attention to the opposite limit of an almost "closed" chaotic system: $T \ll 1$. Exploiting $g \gg 1$ we find for arbitrary number of open channels and arbitrary $y$ the following universal (up to a coefficient) behaviour of delay time distribution:

$$
\mathcal{P}\left(\tau_{s}\right) \propto g^{-1 / 2} \tau_{s}^{-3 / 2} \quad \text { when } \quad g^{-1} \ll \tau_{s} \ll g
$$

in the parametrically large region of delay times. The proportionality coefficient in this formula depends on the symmetry breaking parameter $y$ and $M$ in a complicated way.
Such a $\tau^{-3 / 2}$ behaviour holding irrespective of the TRI symmetry is the most robust feature of the time delay statistics of weakly open chaotic systems. It was first obtained in [18] for the case of broken TRS, but physical arguments show that it is a very general feature simply following from the picture of well-isolated resonances typical for such systems 18 . It also seems to be quite insensitive to the particular details of definitions of time delays and holds for distributions of such slightly different quantities as Wigner-Smith time delay, partial delay times or even "dwell times". In Fig.1 we plotted a typical fluctuating pattern of energy-dependent "dwell times" obtained in the paper [10] in the course of numerical simulations of quantum chaotic scattering on a two dimensional cavity in tunneling contact with two waveguides. Sampling the distribution of dwell times over the chosen range of energies we find a good agreement with the predicted $\tau^{-3 / 2}$ behaviour, see Fig.1.

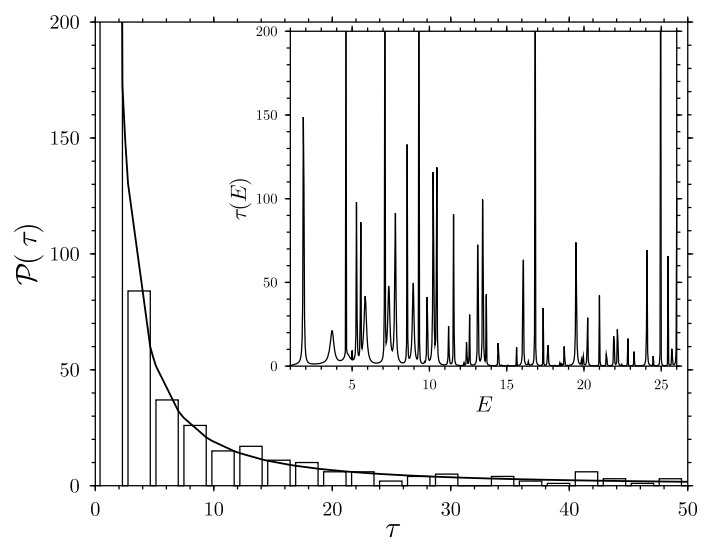

FIG. 1. The distribution of the dwell times $\tau$ in chaotic scattering in weakly open Sinai-like billiard. Inset shows a fluctuating pattern of dwell time versus energy (data were kindly provided to us by the authors of Ref.[10]). Solid line shows the theoretical prediction $\mathcal{P}(\tau) \propto \tau^{-3 / 2}$.

Outside the parametrically large interval $g^{-1} \ll \tau_{s} \ll$ $g$ our general expression Eq.(5) predicts an exponential cutoff at $\tau_{s} \lesssim g^{-1}$ and a crossover to the behaviour described by Eq.(6) for the asymptotically large times $\tau \gg g$. One can check that for "weakly broken" TRI again there emerges a scale $\tau_{y}(g) \sim g / y^{2}$ such that the asymptotic tail is GOE-like at $g \ll \tau_{s} \ll \tau_{y}(g)$, but always GUE like for $\tau_{s} \gg \tau_{y}(g)$, in full agreement with the discussion above.

Finally, having in mind the comparison with the semiclassics let us consider in more detail the large-channel limit $M \gg 1$ of our general expressions like Eq.(2) describing the correlations of phase shifts and WignerSmith time delays. When doing this it is natural to consider the angle difference $\phi=\phi_{1}-\phi_{2}$ to be of the order of $\phi \sim 1 / M \ll 1$. Then one rescales $\phi \equiv \tilde{\phi} / M$, substitutes $\tan \phi \sim \tilde{\phi} / M$ in the "channel factor" Eq.(3) and performs the limit $M \rightarrow \infty$ explicitly. For $\omega=0$ the re- 
sulting expression turns out to be identical to the parametric correlation function of eigenvalues of large random matrices in the crossover regime derived for the first time by N.Taniguchi et al. 25]. Taking into account that $\phi_{1}-\phi_{2} \sim 1 / M$ results also in $\theta_{1}-\theta_{2} \sim 1 / M$, we conclude that the statistics of scattering phase shifts in the large $M$ limit is just the same as that of energy levels of closed chaotic systems. The latter conclusion is in agreement with the available numerical results obtained for a realistic model of chaotic systems with $M=23$ in [9]. It is also interesting, that the only modification required for $\omega \neq 0$ is to replace $\tilde{\phi} \rightarrow \tilde{\phi}+\omega$.

Considering the time delay characteristics one should take into account that the width of the time delay distribution is of lower order in $M$ as compared with the mean value $\left\langle\tau_{W}\right\rangle$ when resonances are overlapping: $M T \gg 1$ [19. To extract the time delay correlations in the corresponding limit requires a calculation similar to that done in the paper by Pluhar et al. [25]. The resulting expression turns out to be quite a transparent one and is given by:

$$
K_{\omega, x, y_{1}, y_{2}}^{\tau}=\frac{1}{2}\left(\frac{\Gamma_{-}^{2}-\omega^{2}}{\left[\Gamma_{-}^{2}+\omega^{2}\right]^{2}}+\frac{\Gamma_{+}^{2}-\omega^{2}}{\left[\Gamma_{+}^{2}+\omega^{2}\right]^{2}}\right)
$$

provided that $\Gamma_{ \pm} \equiv M T / 2+x^{2}+\left(y_{1} \pm y_{2}\right)^{2} \gg 1$. Actually, this formula is nothing else but the semiclassical expression for the time delay correlator. It can be obtained from the Gutzwiller trace formula in diagonal approximation, with the quantity $M T / 2$ being replaced by the decay rate out of the chaotic region, see 114,18.

The last point to be mentioned is related to the issue of fluctuations of low-frequency admittance as defined in 13. We noted above that the time delay fluctuates weakly in the many-channel limit $M \rightarrow \infty$. Using this fact and the relation between the time delay and the low-frequency admittance $G^{I}(\omega)$ presented in [13] (see, however, 26]) one finds that the parametric correlator $\left\langle G^{I}\left(0, y_{1}\right) G^{I}\left(x, y_{2}\right)\right\rangle /\left\langle G^{I}\right\rangle^{2}-1$ of the admittance in the limit $M \gg 1$ is given by:

$$
\frac{1}{2}\left(\frac{1}{\Gamma_{-}^{2}}+\frac{1}{\Gamma_{+}^{2}}\right)\left(1+\frac{M e^{2}}{C_{e} \Delta}\left\langle\tau_{W}\right\rangle\right)^{-2}
$$

where $C_{e}$ denotes the so called "geometric capacitance" [13] and $e$ stands for the electron charge. In the limiting cases of unbroken $\left(y_{1}=y_{2}=0\right)$ and completely broken $\left(y_{1}=y_{2} \rightarrow \infty\right)$ TRI this expression coincides with that found recently by another method by Brouwer and Büttiker 13.

We are very greatful to V.V. Sokolov for instructive discussions and to Prof. Hong Guo for kindly providing us with the numerical data used to extract the time delay distribution depicted in Fig.1. The financial support from SFB 237 der Deutschen Forschungsgemeinschaft as well as from the program "Quantum Chaos" (grant No. INTAS-94-2058) is acknowledged with thanks.
[1] E. Wigner, Phys. Rev. 98 (1955) 145; F.T. Smith, Phys. Rev. 118 (1960) 349; ibid 119 (1960) 2098.

[2] E.Akkermans et al., Phys.Rev.Lett. 66, 76 (1991);

[3] B.L.Altshuler and B.D.Simon in: Mesoscopic Quantum Physics. Proceedings of the Les-Houches Summer School. Session LXI ed. by E.Akkermans et al,( Elsever, 1995),p.5

[4] U.Smilansky in Chaos and Quantum Physics. Proceedings of the Les-Houches Summer School. Session LII, ed. by M.J.Giannoni et.al (North Holland, Amsterdam, 1991),p.372

[5] P.Gaspard in: Quantum Chaos. Proceedings of E. Fermi Summer School, ed.by G.Casati et.al. (1991), p.307

[6] E.Doron, U.Smilansky and A.Frenkel, Phys.Rev.Lett. 65,3072 (1990) and in 周, p.399.

[7] A.Shushin and D.W.Wardlaw, J.Phys.A:Math.Gen. 25, 1503 (1992)

[8] R.A.Jalabert, J.-L.Pichard, J. Phys.I France 5 ,287 (1995); E.Mucciolo, R.A.Jalabert,J.-L.Pichard and B.L.Altshuler , unpublished

[9] B.Dietz, M.Lombardi, T.H.Seligman, Phys.Lett.A 215,181 (1996)

[10] Y.Wang, N.Zhu, J.Wang and H.Guo, Phys.Rev.B 53, 16408 (1996)

[11] P.W.Anderson, P.A.Lee Suppl.Prog.Theor.Phys. 69 (1980), 212

[12] E.Buks et al.Phys.Rev.Lett.77 (1996),4664

[13] V.Gopar,P.Mello and M.Büttiker , Phys.Rev.Lett. 77 3005 (1996); P.Brouwer and M.Büttiker cond-mat 9610144

[14] B.Eckhardt, Chaos 3 , 613(1993)

[15] J.J.M. Verbaarschot, H.A. Weidenmüller, and M.R. Zirnbauer, Phys. Rep. 129 (1985) 367.

[16] K.B. Efetov, Adv. Phys. 32 (1983) 53.

[17] Y.V. Fyodorov and H.-J. Sommers, Phys. Rev. Let. 76 (1996) 4709 and JETP Lett. 63 (1996),1026

[18] Y.V. Fyodorov and H.-J. Sommers, cond-mat/970103

[19] N. Lehmann, D.V. Savin, V.V. Sokolov, and H.-J. Sommers, Physica D 86 (1995) 572

[20] H.L. Harney, F.M.Dittes and A. Müller, Ann. Phys. (NY) 220 (1992) 159.

[21] P.Seba, K.Zyczkowski and J.Zakrzewski, Phys.Rev.E 54 (1996), 2438

[22] A.M.S. Macedo, Phys.Rev. E 50,R659 (1994)

[23] A.Pandey, M.L.Mehta Commun.Math.Phys. 16 (1983), 2655; J.B.French et al. Ann.Phys. 181 (1988),198

[24] N.Dupius and G.Montambaux Phys.Rev.B 43 (1991),14390; K.Zyczkowski and G.Lenz Z.Phys.B 82 (1991), 299; H.-J.Sommers and S.Iida Phys.Rev.E 49 (1994); V.I.Fal'ko and K.B.Efetov Phys.Rev.B 50 (1994) and Phys.Rev.Lett. 77(1996),912; O.Bohigas et al. Nonlinearity 8, 203(1995); Y.Alhassid et al. condmat/9609115; van Langen et.al. Phys.Rev.E 55 (1997),1

[25] A.Altland et al. Ann.Phys. 219(1992), 148; N.Taniguchi et al. Europh.Lett. 27 (1994), 335 A.Altland et al. J.Phys.A:Math.Gen 26 (1993),3545; Z.Pluhar et al , Ann.Phys.(NY) 243,1 (1995)

[26] The effects of charge quantization not taken into account in 13 may lead to modification of the results, see I.L.Aleiner and L.I.Glazman cond-mat/9612138. . 